Western University

Scholarship@Western

$1-2014$

\title{
Brain metastases from breast cancer: lessons from experimental magnetic resonance imaging studies and clinical implications.
}

Donna Murrell

The University of Western Ontario, dmurrel@uwo.ca

Paula J Foster

Western University, pfoster4@uwo.ca

Ann F Chambers

Western University, ann.chambers@lhsc.on.ca

Follow this and additional works at: https://ir.lib.uwo.ca/biophysicspub

Part of the Medical Biophysics Commons

\section{Citation of this paper:}

Murrell, Donna; Foster, Paula J; and Chambers, Ann F, "Brain metastases from breast cancer: lessons from experimental magnetic resonance imaging studies and clinical implications." (2014). Medical Biophysics Publications. 36.

https://ir.lib.uwo.ca/biophysicspub/36 


\title{
Brain metastases from breast cancer: lessons from experimental magnetic resonance imaging studies and clinical implications
}

\author{
Donna H. Murrell • Paula J. Foster • Ann F. Chambers
}

Received: 31 October 2013 / Revised: 14 November 2013 / Accepted: 19 November 2013 / Published online: 5 December 2013

(C) Springer-Verlag Berlin Heidelberg 2013

\begin{abstract}
Breast cancer that has metastasized to the brain presents difficult clinical challenges. This diagnosis comes with high mortality rates, largely due to complexities in early detection and ineffective therapies associated with both dormancy and impermeability of the blood-brain barrier (BBB). Magnetic resonance imaging (MRI) is the current gold standard for diagnosis and assessment of brain tumors. It has been used clinically to investigate metastatic development as well as monitor response to therapy. Here, we describe preclinical imaging strategies that we have used to study the development of brain metastases due to breast cancer. Using this approach, we have identified three subsets of metastatic disease: permeable metastases, nonpermeable metastases, and solitary, dormant cancer cells, which likely have very different biology and responses to therapy. The ability to simultaneously monitor the spatial and temporal distribution of dormant cancer cells, metastatic growth, and associated tumor permeability can provide great insight into factors that contribute to malignant proliferation. Our preclinical findings suggest that standard clinical detection strategies may underestimate the true metastatic burden of breast cancer that has metastasized to the brain. A better understanding of true metastatic burden in brains will be important to assist in the development of more effective chemotherapeutics - particularly those targeted to
\end{abstract}

D. H. Murrell • P. J. Foster · A. F. Chambers

Department of Medical Biophysics, Western University,

London, ON, Canada

D. H. Murrell • P. J. Foster

Imaging Research Laboratories, Robarts Research Institute,

London, ON, Canada

A. F. Chambers $(\bowtie)$

London Regional Cancer Program, 790 Commissioners Road East,

London, ON, Canada N6A 4 L6

e-mail: ann.chambers@Lhsc.on.ca cross the $\mathrm{BBB}$ - as well as detection of small nonpermeable metastases.

Keywords Breast cancer · Metastasis · Dormancy · MRI · Iron · Nanoparticles

\section{Introduction}

Breast cancer metastasis to the brain is a serious and increasing medical problem. In this review, we will discuss the clinical needs for better understanding of metastases of breast cancer to the brain and discuss studies in which novel preclinical magnetic resonance imaging (MRI) approaches are shedding light on this clinically important problem.

\section{Breast cancer and metastasis}

Breast cancer is the most common cancer (excluding nonmelanoma skin cancers) in both American and Canadian women [1, 2], with 232,340 new cases expected in the USA [3] and 23,800 in Canada [2] this year alone. Early detection and improvements in both screening technology and systemic treatment are believed to have contributed to a $42 \%$ decrease in mortality rate in Canada since its peak in 1986; the agestandardized mortality rate has declined from 32 deaths per 100,000 in 1986 to 18.7 deaths expected per 100,000 in 2013 $[1,2]$. In the USA, it is estimated that 39,620 women will die from this disease in 2013 [3]. These mortality rates have been declining since the 1990s and are currently the lowest in Canada since 1950 similar trends are being seen worldwide. Despite this trend, breast cancer is still the second leading cause of cancer death in both American and Canadian women $[1,2]$. The relatively high mortality rate is primarily attributed to the propensity of breast tumors to metastasize to regional and distant sites, where current systemic treatments, such as 
chemo-, hormone, and targeted therapies ultimately fail. Common locations for breast cancer metastases to develop are the lung, liver, bone and brain $[4,5]$.

Cancer metastasis is an inefficient process [4, 6]. High numbers of circulating cancer cells are often present in blood samples from cancer patients; however, few actual metastatic tumors result $[7,8]$. This suggests that cancer cell survival during the metastatic cascade must be low, or conditions for cell proliferation in the microenvironment are highly specific. The metastatic cascade begins with primary cancer cells gaining the ability to migrate and invade the surrounding stroma and then intravasate into nearby vasculature. They must survive in the circulation until their eventual arrest at a distant organ; from here, cells extravasate from the blood vessel into the surrounding tissue. Studies indicate that cells survive both the intravasation and extravasation processes with high efficiency; however, a cell's ability to thrive once at a distant site is much less efficient $[4,9]$.

\section{Brain metastases of breast cancer}

Brain metastasis is a terrifying diagnosis and occurs in 20 $30 \%$ of metastatic breast cancer patients [10]. The incidence of brain metastases is increasing, which is believed to be due to the introduction of more sensitive diagnostic methods, improved systemic therapies leading to improvements in extracranial control and survival, and more frequent use of screening studies [11, 12]. Brain metastasis can occur in patients with all subtypes of breast cancer; however, several factors have been identified that put particular breast cancer patients at higher risk. Risk of brain metastasis is high for patients who (1) are young, (2) have lymph node-positive disease and/or other systemic metastases, (3) are diagnosed with "triple-negative" breast cancer (ER-, PR-, HER2 unamplified), and/or whose tumors (4) overexpress HER2 [13, 14]. In patients with the HER2-positive metastatic breast cancer, the frequency of brain metastasis has been reported to be as high as $50 \%$ [15]. Brain metastases have emerged as a major challenge affecting morbidity and mortality of patients with HER2-positive metastatic breast cancer [16].

\section{Dormancy}

Once a cancer cell spreads to a distant site, it may experience one of three fates: (1) it may die, (2) it may begin to proliferate, or (3) it may remain viable but dormant ("nonproliferative") [4, $9,17]$. If cancer cells survive in the metastatic organ, they may coexist in three forms. Solitary cells may remain in a state of quiescence whereby they remain viable but nonproliferative for an extended period of time; this process of dormancy is likely responsible for clinical recurrence. If solitary cells begin to proliferate, they may form "dormant" micrometastasesproliferative tumors where net tumor size is unchanged due to balanced proliferation and apoptosis - or they may become actively growing and recruit vasculature by either angiogenic or vessel co-option strategies. These three states of existence represent vastly different cancer biology with significant implications for detection, therapy, and patient outcomes. The factors that control the balance between dormancy and proliferation are poorly understood but are subject of much ongoing research [18-21].

Dormancy, both at the single cell level as well as at the micrometastasis stage, is believed to provide a reservoir of cells that lie in wait until some elusive trigger causes them to proliferate and progress into clinically relevant tumors. This phenomenon presents a significant treatment challenge because its biology deviates from traditional understanding of malignant cancer growth. Current chemotherapies target highly proliferating cells and therefore dormant cells persist unaffected [22, 23].

\section{Treatment of brain metastases}

Current therapies for brain metastases include steroids, surgical excision of solitary or small numbers of metastases (oligometastatic disease), stereotactic radiosurgery for small $(<3 \mathrm{~cm})$ lesions not amenable to surgery, and whole brain radiotherapy (WBRT). These therapies are considered to be palliative, rather than curative. Optimization of these therapies and combination treatment strategies have improved prognosis; however, the median time from diagnosis of brain metastasis to death is still grim at 4-6 months, and only $20-40 \%$ of patients are alive at 1 year [24].

Unique features of brain metastases complicate treatment. Most chemo- and molecular systemic therapies are ineffective against brain metastases, due to their inability to cross the intact blood-brain barrier (BBB). The BBB is a combination of endothelial cells, pericytes, and astrocytes; under normal circumstances, they act together to protect the brain by controlling the passage of substrates from the blood into the brain parenchyma. In the local area of a tumor, this is sometimes referred to as the blood-tumor barrier (BTB). A prime example is the monoclonal antibody trastuzumab $\left(\right.$ Herceptin $\left.^{\circledR}\right)$. Trastuzumab inhibits the growth of a variety of cancer cells overexpressing HER2 in cell culture, animal models, and clinical trials [25-28]. Its success in clinical trials with breast cancer led to US FDA approval for its use in the treatment of metastatic breast cancer when combined with chemotherapy. Many studies have reported high rates of central nervous system (CNS) recurrences in HER2-positive breast cancer patients treated with trastuzumab [29-31]. Typically, brain metastases manifest while systemic metastases are still responding to trastuzumab-containing regimens. In fact, trastuzumab prolongs overall survival in HER2+ patients with brain metastases. This type of mixed response is due to the fact that trastuzumab cannot cross the BTB; hence, its role is limited to controlling extra-CNS metastases [32]. 
Management of breast cancer metastasis is further complicated by high recurrence rates, sometimes even decades after apparent successful treatment of the primary tumor. Even if patients initially respond to WBRT, about half will experience recurrence in the brain within 1 year [33] either at the site of previous lesions, or elsewhere.

Early detection and treatment of breast cancer - prior to the dissemination of cancer cells to distant sites and start of metastatic growth - is associated with favorable outcomes and high probability of cure or long-term survival. On the contrary, viable treatment options become limited, and prognosis is poor, if cancer is identified after it has metastasized. Therefore, the ability to detect cancer and metastasis at the earliest steps is paramount to patient survival. MRI provides an ideal means to detect and monitor brain metastases due its high resolution and good soft tissue contrast without the use of ionizing radiation.

\section{Magnetic resonance imaging in the study of metastasis in the brain}

Clinical MRI for brain metastases

For the clinical evaluation of brain metastases, MRI is currently the gold standard. Gadolinium-DTPA (Gad) contrastenhanced MRI is widely regarded as the most accurate diagnostic imaging modality for brain tumors [34]. Gad has a molecular weight of $590 \mathrm{Da}$ and is routinely used in MRI to identify BBB breakdown [35]. In patients, MRI is performed before and after intravenous (IV) Gad. In brain tumors, Gad leaks from the vascular system into the interstitial space only when the BBB is disrupted, that is, when the tumor increases the permeability of preexisting blood vessels or induces the growth of leaky blood vessels [36]. This is indicated by an

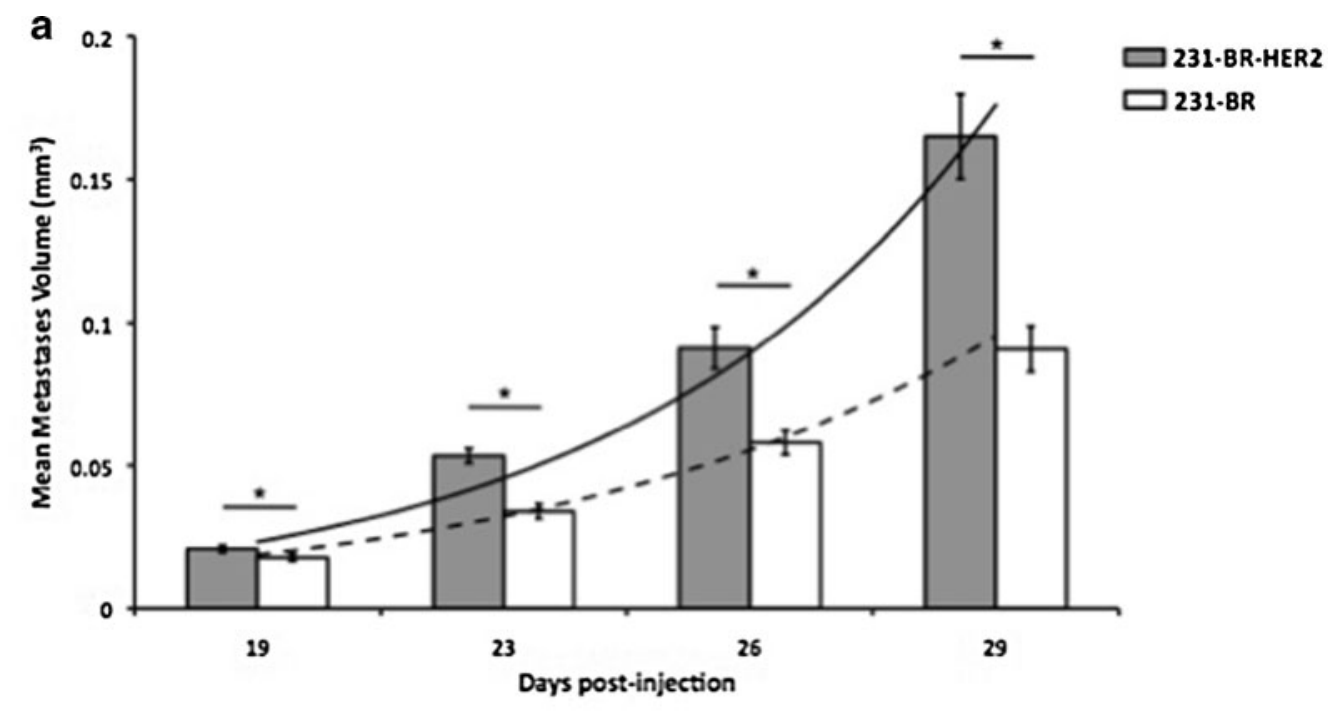

b

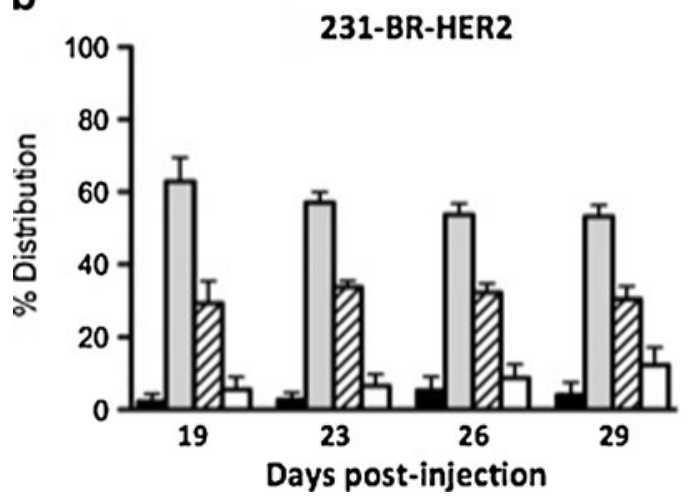

Fig. 1 Characterization by MRI of two human breast cancer cell lines producing brain metastases in mice. a Mean metastases volumes in the whole brain for MDA-MB-231-BR-HER2 and MDA-MB-231-BR cells. The mean volume was significantly greater for 231-BR-HER2 mice (gray bars) compared with 231-BR mice (white bars) at every time point ( ${ }^{*} P<$ $0.05)$. The growth rates were $67.5 \%$, with $R^{2}=0.98$ for 231-BR-HER2 metastases (solid line) and $54.4 \%$ with $R^{2}=0.99$ for $231-\mathrm{BR}$ metastases

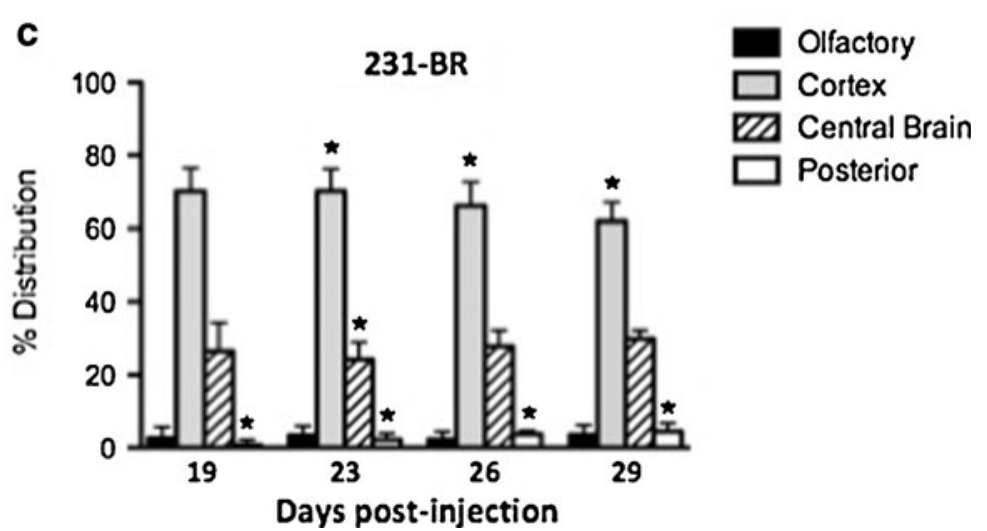

(dashed line). b, c Relative distribution of metastases in the brain for 231-BR-HER2 and 231-BR mice. The percent distribution of tumors in each brain region is shown for all time points for 231-BR-HER2 mice (b) and 231-BR mice (c). The asterisks denote a statistically significant difference $(P<0.05)$ between the two cell lines. Adapted with permission from Perera et al. [45] 
increase in signal intensity (enhancement) of the tumor in the post-Gad image.

Since longitudinal imaging can be performed with MRI, it provides an excellent tool for investigating tumor growth and responses to treatment over time. Many standard MRI pulse sequences have been applied to study brain tumors clinically; these include anatomical MRI, dynamic contrast-enhanced MRI-this provides information about tumor vasculature [37], and diffusion-weighted MRI [38] — where water diffusion patterns are related to tissue structure at the molecular level. The combination of these MRI techniques and can reveal characteristics of normal tissue and highlight important information about disease states.

Imaging the development of brain metastases due to breast cancer in preclinical models

To study breast cancer metastasis to the brain, mouse models have been developed using human breast cancer cell lines [39-42]. Only a handful of models specific to breast cancer brain metastasis have been described. A physiologically relevant and reliable model system for studying metastasis should include as many of the steps in the metastatic cascade as possible. The best-characterized models for brain metastases utilize the direct injection of cells into the left cardiac ventricle $[40,41]$. Cells injected by this route will be delivered to many organs via the arterial blood flow, and about 3.5-9.5\% of these cells will be delivered to the brain, due to cardiac output $[43,44]$, where they will have the opportunity to colonize if there is a match between the cells' growth requirements and the microenvironment of the brain. In preclinical studies, brain metastases have been characterized mainly by histology, immunohistochemistry, and fluorescence microscopy in these models. These techniques allow for measurements of the numbers of metastases and the cross-sectional area of metastases and for an analysis of cellular markers. Although these methods are able to provide detailed molecular and morphologic information, they are limited to ex vivo studies, and only an end-point analysis is permitted. Due to its high spatial resolution, sensitivity, noninvasive nature, and diverse set of image contrast possibilities, MRI provides an ideal opportunity to study the dynamic process of cancer and brain metastasis in vivo.

A wealth of information can be derived about the development of metastases from in vivo three-dimensional (3D) MRI. MRI permits multiple scanning sessions to be performed to monitor the temporal features of metastasis, whereas histology only permits a single snapshot in time. MR images can also be acquired in three dimensions, allowing the whole brain to be analyzed in all spatial orientations and without gaps between slices; this is unlike histologic analyses where it is a practical impossibility to analyze the whole brain, section by section. Metastases volumes can be measured from 3D MRI, which represent their size in vivo, although similar measurements taken from histologic tissue sections must account for shrinkage and deformation due to the chemical processing. The analysis of volumes over time can provide information about growth rates for individual metastases. Overall, MRI can provide a much more complete picture of brain metastasis than can traditional histology.

3D MRI has been used to monitor the spatial and temporal development of brain metastases in the whole mouse brain. Perera et al. used MRI to compare the growth of 231BR and 231BR-HER2 brain metastases [45]. The number of

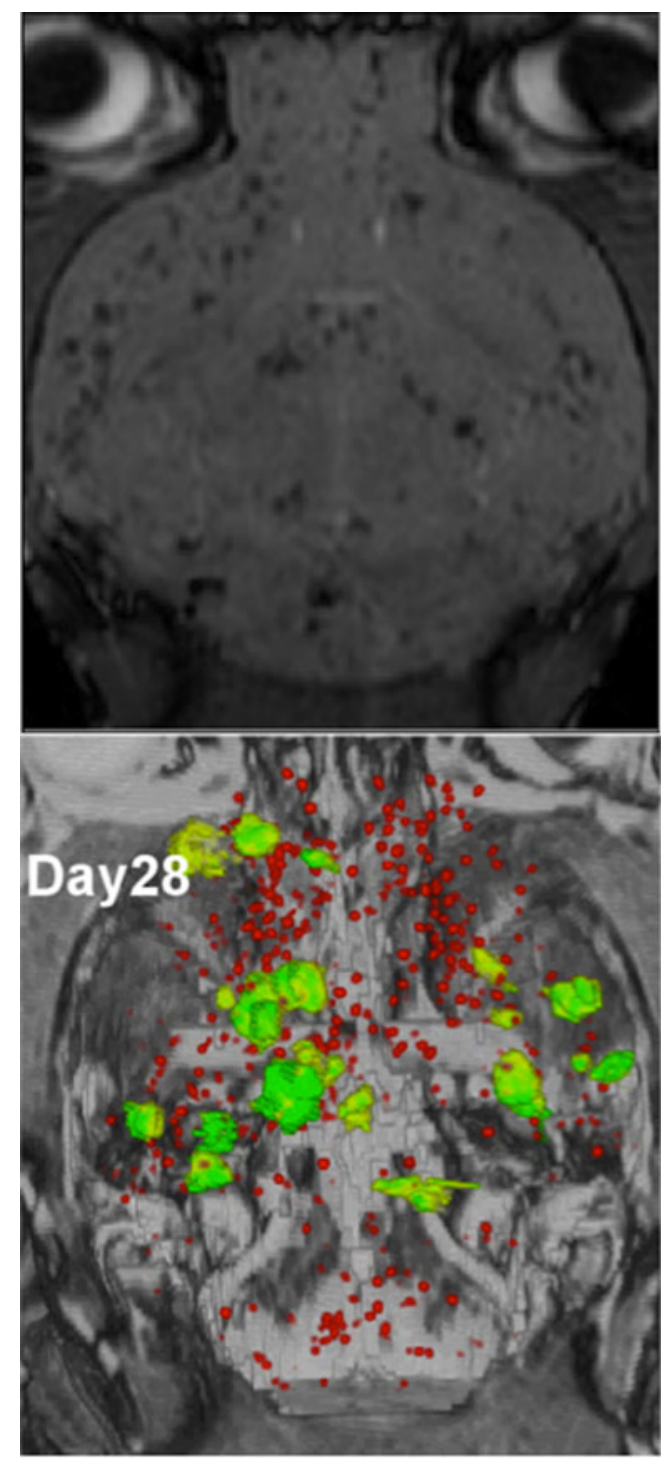

Fig. 2 Detection of iron-labeled breast cancer cells in the mouse brain by MRI. 100,000 iron-labeled MDA-MB-231BR/EGFP cells were injected into the left ventricle of the heart for delivery to the brain, using procedures as described [50]. Image acquired on day 0 (top) shows hypointensities (dark spots) that correspond to single cells and small clusters of cells. On day 28 (bottom), a surface rending of the cells and tumors in the brain at the endpoint of the experiment is presented. The brain metastases are indicated in green. The iron-retaining, nonproliferative cancer cells that persist in the brain are indicated in red 
metastases and their volumes were quantified over the entire brain of each mouse at multiple time points. 231BR-HER2 cells produced the same numbers of metastases as 231BR but they were significantly larger. The growth rate for 231-BRHER 2 metastases was $67.5 \%$ compared with $54.4 \%$ for the 231-BR metastases. More than $50 \%$ of metastases were located in the cortex and 25 to $30 \%$ of metastases were identified in the central brain for each time point and for mice injected with either cell line (Fig. 1).

Imaging dormant cancer cells

Nonproliferative cancer cells have been imaged in vivo using a novel procedure referred to as cellular MRI. Cellular MRI is achieved by pre-labeling cancer cells with iron oxide nanoparticles prior to their administration. The presence of intracellular iron causes a distortion in the local magnetic field resulting in abnormal signal hypointensity in iron-sensitive images. Therefore, iron-labeled cells appear as regions of signal loss or black holes in the MR images. Heyn et al. have demonstrated that even single iron-labeled cells can be detected in vivo [46].

Cellular MRI is most often employed to image cells after their transplantation. For example, many different types of stem and progenitor cells have been successfully tracked in vivo with MRI using iron oxide and studies show there is minimal impact on cell function or phenotype at a wide range of iron loading levels [47-49]. Very few groups have used cellular MRI to study cancer cells because the iron label is

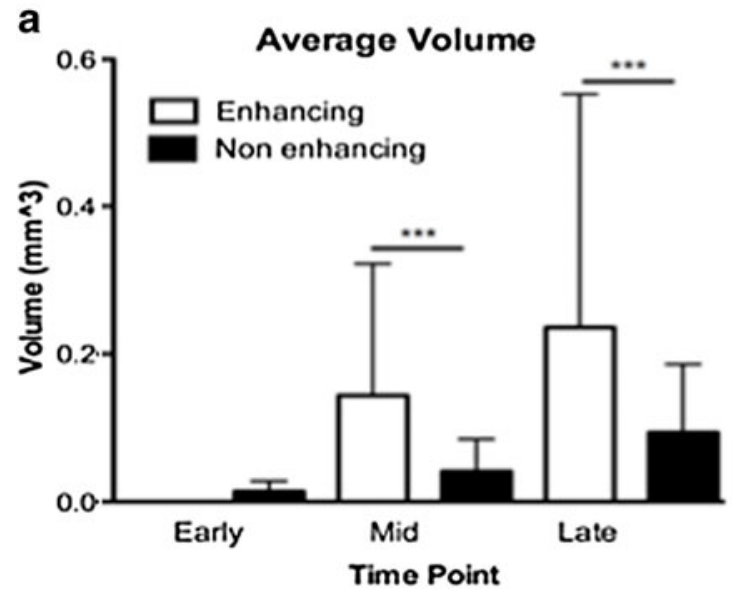

C
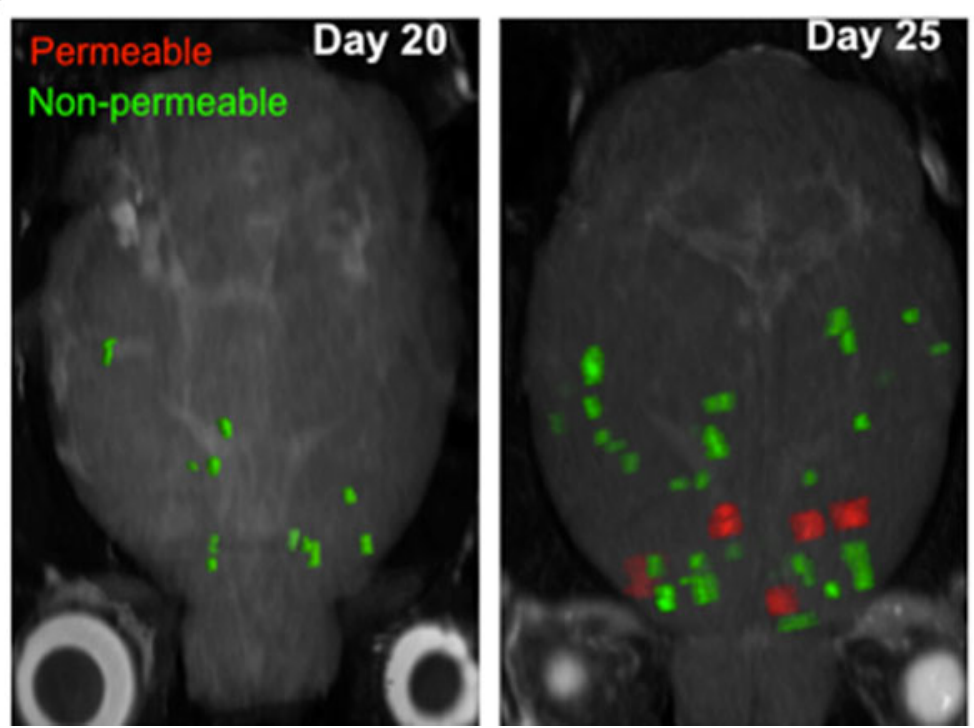

\section{Metastasis Volumes}
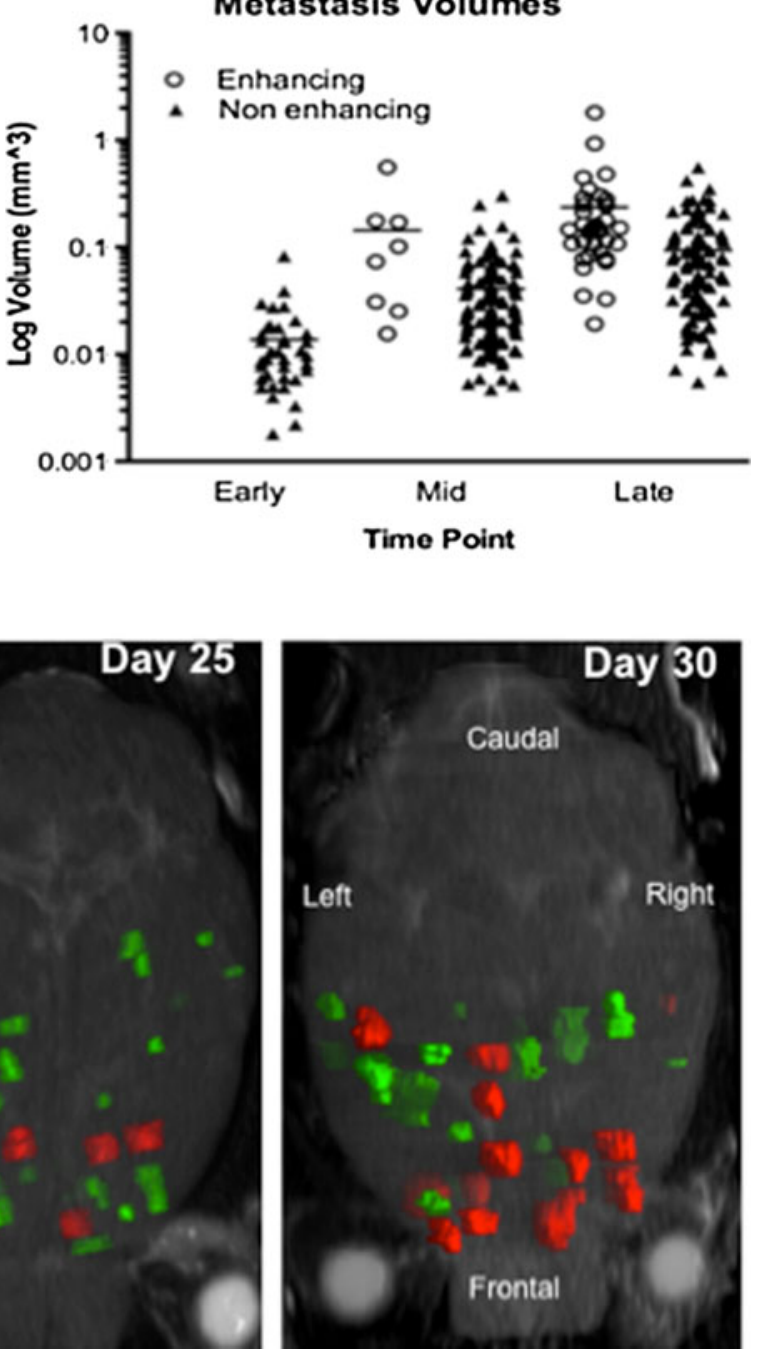

Fig. 3 Volume measurements of Gad-enhancing and nonenhancing brain metastases on post-Gad T1-weighted spin echo images in mice. a At both mid and late time points, the average volume of enhancing metastases (those appearing hyperintense/white on the post-Gad images) was significantly larger than nonenhancing metastases $(P<0.05)$. However, there was a wide range of volumes for both enhancing and nonenhancing metastases (b). There also appeared to be a minimum volume threshold of enhancing metastases, although being larger than this did not guarantee enhancement. c 3D volume rendering of a mouse brain in the coronal plane, from the same mouse at each time point. Gad-enhancing metastases are rendered in red and nonenhancing metastases are shown in green. Neither volume nor position in the brain appears to have an influence on whether metastasis was enhanced or not. Adapted with permission from Percy et al. [53] 
diluted over time in proliferative cells, leading to loss of label and therefore loss of cell detection. However, cancer cells that are nonproliferative, or slowly cycling, will retain the iron label and the fate of these cells can be tracked with MRI.

Heyn et al. were the first to identify nonproliferative cancer cells in the brain using iron oxide nanoparticles [50]. MRI was used to follow the fate of 231BR cells labeled with micronsized iron particles (MPIO) for 1 month; images were obtained on days 0,4 , and 7 and then weekly. MPIO-labeled solitary cells, and small clusters of cells, were identified as regions of signal void in the mouse brains. At the experimental endpoint (day 28 post cell injection), there were three distinct cell fates (Fig. 2). There was a population of cells that was called "transient" (94\%); these were discrete signal voids in images on day 0 that disappeared over time (usually between days 0 and 7). There was a population of cells that were called "proliferative" (1.5\%); in images acquired on day 0 , these cells appeared as discrete regions of signal loss, but they were replaced by a tumor in images on day 28 . Finally, there was a population of cells that was referred to as "nonproliferative" (4.5\%); these were discrete signal voids in images on day 0 that appeared unchanged on day 28 .

\section{Imaging the integrity of the blood-tumor barrier}

The integrity of the local BBB, also referred to as the BTB, can be compromised in the presence of a tumor and become increasingly permeable. Several studies have now shown that there is considerable heterogeneity in the permeability of individual brain metastases; however, little is known about how or when brain metastases become more permeable [51, 52]. Percy and colleagues used Gad-enhanced MRI to track the development of brain metastases due to breast cancer in a mouse model [53]. At early time points, small metastases were uniformly nonpermeable. Many of the metastases became permeable with time, suggesting that as they develop, changes to the tumor vasculature compromise the integrity of the BBB. At the last imaging time point, there were approximately four times as many Gad-permeable metastases as nonpermeable. Gad-permeable metastases were significantly larger than nonpermeable tumors; however, size alone was not sufficient to predict permeability (Fig. 3).

The impermeability of the BBB hinders the delivery of imaging contrast agents and systemic therapies to the brain; this raises two important issues to consider for clinical translation. First, at early time points, small metastases in the mouse model were uniformly nonpermeable [53]; this suggests that some brain metastases in patients may currently be undetectable on typical T1w MR images and patient tumor burden is not being accurately assessed. Secondly, the intact BBB prevents most chemo- or molecular-targeted therapies from reaching these tumors. MRI offers a means to noninvasively assess the permeability status of brain metastases, which could be important for understanding the process of true metastatic burden at early time points and for evaluating the development and monitoring the effectiveness of BBBpermeable chemotherapeutic drugs.

\section{Limitations of MRI to study brain metastasis}

MRI has some limitations for preclinical investigations of brain metastases. For anatomical imaging, there is a limit to the spatial resolution that can be achieved, even with dedicated animal imaging systems. As a result, micrometastases, which can be readily detected by traditional histological methods, can be missed in MR images. The cost for performing longitudinal MRI studies can also be limiting. MRI systems are expensive and are typically operated by cost recovery, meaning that scanning is charged hourly. In vivo MRI of mice requires scanning sessions on the order of hours. Other imaging modalities, such as optical imaging, are much less expensive and allow for high throughput imaging but have very limited depth penetration.

Cellular MRI also has drawbacks and concerns. For one thing, there are other sources of signal loss in images that are sensitive to iron (for example, the bones, air, and blood products associated with hemorrhage), making it difficult to unambiguously identify regions containing labeled cells. In
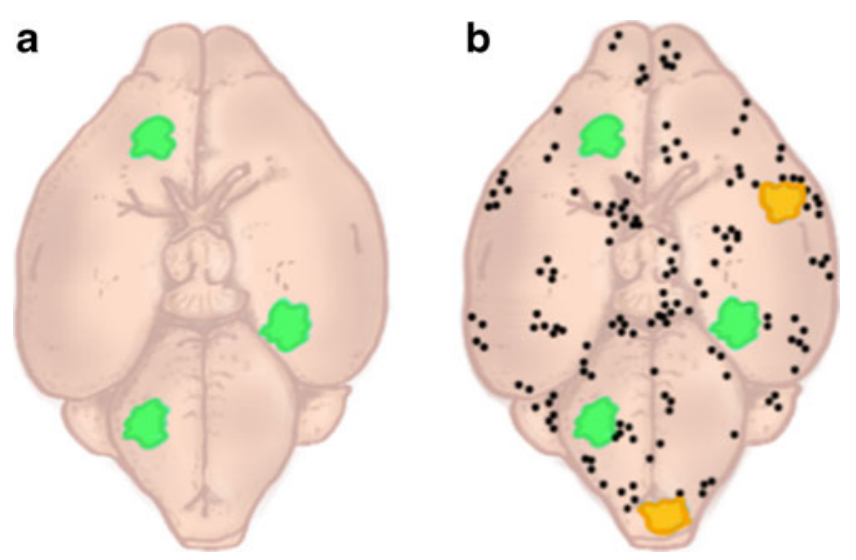

Fig. 4 Schematics of brain metastatic burden due to breast cancer. In a, the green-colored brain tumors represent those brain metastases that are detected by Gad-enhanced MRI; these metastases have a compromised blood-tumor barrier. In $\mathbf{b}$, these metastases are again shown in green along with (1) yellow-colored brain tumors that are detectable by MRI using the bSSFP sequence (these metastases do not have a disrupted BTB and therefore are not detected by Gadolinium-enhanced MRI) and (2) cpo regions of signal void that represent nonproliferative or dormant cancer cells which may be present in the brain but which also would go undetected by conventional MRI techniques. $\mathbf{b}$ is based on our preclinical findings reviewed here. Thus, current clinical assessment of metastatic burden in brain in breast cancer patients using Gad-enhanced MRI may provide an underestimate of true metastatic burden. Clinical management decisions may be quite different depending on the ability to detect true metastatic burden in brain. Additionally, the three categories of metastatic burden depicted in $\mathbf{b}$ represent distinct therapeutic targets, for which new therapeutic approaches are needed 
addition, iron-labeled cell quantification is difficult. A number of groups have shown that the contrast generated by ironlabeled cells increases with the amount of iron/voxel but this is only linear at low iron loadings and the change in contrast reaches a saturation plateau at higher iron loadings. When quantifying the presence of iron-labeled cells over time, most studies measure the "signal void volume" or the "number of black pixels" and present the change relative to the first imaging time point.

\section{Conclusions and clinical implications}

The metastatic burden in the brain as detected clinically by current methods may significantly underestimate the true metastatic burden (Fig. 4a). Gad-enhancing metastases can be detected by current, gold standard contrast-enhancing MRI procedures. However, within the brain, there may also exist a subset of macroscopic metastases that do not take up the contrast agent and therefore would go undetected (Fig. 4b). Furthermore, there may be a population of dormant, solitary cancer cells present in the brain (Fig. 4b). These two categories of cancer cells represent distinct therapeutic targets and may be spared by therapies that target permeable metastases and actively dividing cancer cells, leading to later disease recurrences. This poses a challenge for effective cancer detection and therapy [54].

The best treatment for oligometastases is the subject of ongoing debate [55-58]. In some cases, focal treatment of oligometastatic disease (surgery, stereotactic radiosurgery) of single/few metastases prolongs overall or progression-free survival, while in other cases, disease progression occurs often at sites removed from the treated lesions. These recurrences are presumably due to undetected metastatic burden, either as nonenhancing macroscopic lesions or dormant solitary cancer cells. In order to advance therapeutic treatment options, we need improved methods to detect true metastatic burden in patients and a better understanding of the biology of different subsets of metastatic disease - such as dormancy and variance in tumor permeability. Translating this knowledge from preclinical experiments to the clinical setting is vital to understanding metastatic growth, recurrence, and patient survival.

\footnotetext{
Acknowledgments We thank Chelsey Gareau for preparation of Fig. 4. Work described in this review is supported by a grant from the US Department of Defense Breast Cancer Research Program (\#W81XWH06-2-0033). DBP is supported by studentships from the Canadian Institutes of Health Research Strategic Training Program in Cancer Research and Technology and the Translational Breast Cancer Research Unit of the London Regional Cancer Program. AFC is Canada Research Chair in Oncology and receives salary support from the Canada Research Chairs Program.
}

Disclosure statement The authors have no conflicts to declare.

\section{References}

1. Breast Cancer Facts 2013. http://www.nationalbreastcancer.org/ breast-cancer-facts. Accessed 16 Oct 2013

2. Canadian Cancer Statistics 2013. http://www.cancer.ca. Accessed 3 Sept 2013

3. Siegel R, Naishadham D, Jemal A (2013) Cancer statistics, 2013. CA Cancer J Clin 63(1):11-30

4. Chambers AF, Groom AC, MacDonald IC (2002) Dissemination and growth of cancer cells in metastatic sites. Nat Rev Cancer 2:563-572

5. Maxmen A (2012) The hard facts. Nature 485:S50-S51

6. Weiss L (1990) Metastatic inefficiency. Adv Cancer Res 54:159-211

7. Tarin D, Vass AC, Kettlewell MG, Price JE (1984) Absence of metastatic sequelae during long-term treatment of malignant ascites by peritoneo-venous shunting. A clinic-pathological report. Invas Metast 4:1-12

8. Tarin D, Price JE, Kettlewell MG, Souter RG, Vass AC, Crossley B (1984) Mechanisms of human tumor metastasis studied in patients with peritoneovenous shunts. Cancer Res 44:3584-3592

9. Goss PE, Chambers AF (2010) Does tumour dormancy offer a therapeutic target? Nat Rev Cancer 10(12):871-877

10. Canadian Cancer Statistics 2010. http://www.cancer.ca Accessed Feb 2010

11. Sharma M, Abraham J (2007) CNS metastasis in primary breast cancer. Expert Rev Anticancer Ther 7(11):1561-1566

12. Kirsch DG, Loeffler JS (2005) Brain metastases in patients with breast cancer: new horizons. Clin Breast Cancer 2:115-124

13. Pestalozzi BC, Zahrieh D, Price KN, Holmberg SB, Lindtner J, Collins J et al (2006) Identifying breast cancer patients at risk for central nervous system (CNS) metastases in trials of the International Breast Cancer Study Group (IBCSG). Ann Oncol 17(6):935-944

14. Lin NU, Claus E, Sohl J, Razzak AR, Arnaout A, Winer EP (2008) Sites of distant recurrence and clinical outcomes in patients with metastatic triple-negative breast cancer: high incidence of central nervous system metastases. Cancer 113(10):2638-2645

15. Sperduto PW, Kased N, Roberge D, XU Z, Shanley R, Luo X, Sneed P, Chao ST et al (2012) Effect of tumor subtype on survival and the graded prognostic assessment for patients breast cancer and brain metastases. Int J Radiat Oncol Biol Phys 82(5):2111-2117

16. Vern-Gross TZ, Lawrence JA, Case LD, McMullen KP, Bourland JD, Metheny-Barlow LJ, Ellis TL, Shaw EG et al (2012) Breast cancer subtype affects patterns of failure of brain metastases after treatment with stereotactic radiosurgery. J Neurooncol 110(3):381-388

17. MacDonald IC, Chambers AF (2006) Breast cancer metastasis progression as revealed by intravital videomicroscopy. Expert Rev Anticancer Ther 6:1271-1279

18. Allan AL, Vantyghem SA, Tuck AB, Chambers AF (2006-2007) Tumor dormancy and cancer stem cells: implications for the biology and treatment of breast cancer metastasis. Breast Dis 26:87-89

19. Barkan D, Chambers AF (2011) $\beta 1$-integrin: a potential therapeutic target in the battle against cancer recurrence. Clin Cancer Res 17(23): 7219-7223

20. Ghajar CM, Peinado H, Mori H, Matei IR, Evason KJ, Brazier H, Almeida D, Koller A, Hajjar KA, Stainier DY et al (2013) The perivascular niche regulates breast tumour dormancy. Nat Cell Biol 15(7):807-817

21. Sosa MS, Bragado P, Debnath J, Aguirre-Ghiso JA (2013) Regulation of tumor cell dormancy by tissue microenvironments and autophagy. Adv Exp Med Biol 734:73-89

22. Naumov GN, Townson JL, MacDonald IC, Wilson SM, Bramwell VHC, Groom AC, Chambers AF (2003) Ineffectiveness of doxorubicin treatment on solitary dormant mammary carcinoma cells or latedeveloping metastases. Breast Cancer Res Treat 82(3):199-206

23. Townson JT, Ramadan SS, Simedrea C, Rutt BK, MacDonald IC, Foster PJ, Chambers AF (2009) Three-dimensional imaging and 
quantification of both solitary cells and metastases in whole mouse liver by magnetic resonance imaging. Cancer Res 69(21):8326-8331

24. Ohno S, Ishida M, Kataoka A, Murakami S (2004) Brain metastasis of breast cancer. Breast Cancer 11(1):27-29

25. Mokbel K, Hassanally D (2001) From HER2 to herceptin. Curr Med Res Opin 17(1):51-59

26. Baselga J, Albanell J (2001) Mechanism of action of anti-HER2 monoclonal antibodies. Ann Oncol 12(Supp11):S35-S41

27. Daniele L, Sapino A (2009) Anti-HER2 treatment and breast cancer: state of the art, recent patents and new strategies. Recent Pat Anticancer Drug Discov 4(1):9-18 (10)

28. Gonzalez-Angulo AM, Hortobagyi GN, Esteva FJ (2006) Adjuvant therapy with trastuzumab for HER2/neu-positive breast cancer. Oncologist 11(8):857-867

29. Pieńkowski T, Zielinski CC (2010) Trastuzumab treatment in patients with breast cancer and metastatic CNS disease. Ann Oncol 21(5): 917-924

30. Shmueli E, Wigler N, Inbar M (2004) Central nervous system progression among patients with metastatic breast cancer responding to trastuzumab treatment. Eur J Cancer 40(3):379-382

31. Clayton A, Danson S, Jolly S, Ryder W, Burt P, Stewart A et al (2004) Incidence of cerebral metastases in patients treated with trastuzumab for metastatic breast cancer. Br J Cancer 91:639-643

32. Lower E, Blau R, Bismayer J, Brennan L, Broun R, Dannenman W et al (2001) Increased brain metastasis detected in metastatic breast cancer patients receiving herceptin. Breast Cancer Res Treat 69:271

33. Son CH, Jimenez R, Niemierko A, Loeffler JS, Oh KS, Shih HA (2012) Outcomes after whole brain reirradiation in patients with brain metastases. Int J Radiat Oncol Biol Phys 82(2):e167-e172

34. Anzalone N, Gerevini S, Scotti R, Vezzulli P, Picozzi P (2009) Detection of cerebral metastases on magnetic resonance imaging: intraindividual comparison of gadobutrol with gadopentetate dimeglumine. Acta Radiol 50(8):933-940

35. Runge VM, Ai T, Hao D, Hu X (2011) The developmental history of the use of gadolinium chelates as intravenous contrast media for MRI. Invest Radiol 46(12):807

36. van der Molen AJ, Bellin MF (2008) Extracellular gadolinium-based contrast media. Eur J Radiol 66(2):168-174

37. Padhani AR (2002) Dynamic contrast-enhanced MRI in clinical oncology: current status and future directions. J Magn Reson Imaging 16(4):407-422

38. Schaefer PW, Grant PE, Gonzalez RG (2000) Diffusion-weighted MR imaging of the brain. Radiology 217:331-345

39. Rye PD, Norum L, Olsen DR et al (1996) Brain metastasis model in athymic nude mice using a novel MUC1-secreting human breastcancer cell line, MA11. Int J Cancer 68(5):682-687

40. Yoneda T, Williams PJ, Hiraga T et al (2001) A bone-seeking clone exhibits different biological properties from the MDA-MB-231 parental human breast cancer cells and a brain-seeking clone in vivo and in vitro. J Bone Miner Res 16(8):1486-1495

41. Palmieri D, Bronder JL, Herring JM, Yoneda T, Weil RJ, Stark AM, Kurek R, Vega-Valle E, Feigenbaum L, Halverson D et al (2007) Her2 overexpression increases the metastatic outgrowth of breast cancer cells in the brain. Cancer Res 67(9):4190-4198

42. Tanner M, Kapanen AI, Raheem O, Grenman S, Elo J, Elenius K, Isola J (2004) Characterization of a novel cell line established from a patient with Herceptin-resistant breast cancer. Mol Cancer Ther 3(12):1585-1592

43. Quintana A, Raczka E, Bonaccorsi A (1979) Cardiac output distribution measured with radioactive microspheres in the mouse. Pharmacol Res Commun 11(3):245-252
44. Basse P, Hokland P, Heron I, Hokland M (1988) Fate of tumour cells injected into left ventricle of heart in BALB/c mice: role of natural killer cells. J Natl Cancer Inst 80(9):657-665

45. Perera M, Ribot EJ, Percy DB, McFadden C, Simedrea C, Palmieri D, Chambers AF, Foster PJ (2012) In vivo magnetic resonance imaging for investigating the development and distribution of experimental brain metastases due to breast cancer. Transl Oncol 5(3):1-9

46. Heyn C, Ronald JA, Mackenzie LT, MacDonald IC, Chambers AF, Rutt BK, Foster PJ (2006) In vivo magnetic resonance imaging of single cells in mouse brain with optical validation. Magn Reson Med 55:23-29

47. Gonzales-Lara LE, Xu X, Hofstetrova K, Pniak A, Chen Y, McFadden C, Martinez-Santiesteban FM, Rutt BK, Brown A, Foster PJ (2011) The use of cellular magnetic resonance imaging to track the fate of iron-labelled multipotent stromal cells after direct transplantation in a mouse model of spinal cord injury. Mol Imaging Biol 13(4):702-711

48. Noad J, Gonzales-Lara LE, Broughton HC, McFadden C, Chen Y, Hess DA, Foster PJ (2013) MRI tracking of transplanted iron-labeled mesenchymal stromal cells in an immune-compromised mouse model of critical limb ischemia. NMR Biomed 26(4):458-467

49. Lu S, Liu S, Zu Q, Xu X, Yu J, Wang J, Zhang Y, Shi H (2013) In vivo MR imaging of intraarterially delivered magnetically labeled mesenchymal stem cells in a canine stroke model. PLoS ONE 8(2): e54963

50. Heyn C, Ronald JA, Ramadan SS, Snir JA, Barry AM, MacKenzie LT, Mikulis DJ, Palmieri D, Bronder JL, Steeg PS et al (2006) In vivo MRI of cancer cell fate at the single-cell level in a mouse model of breast cancer metastasis to the brain. Magn Reson Med 56:1001-1010

51. Zhang RD, Price JE, Fujimaki Tet al (1992) Differential permeability of the blood-brain barrier in experimental brain metastases produced by human neoplasms implanted into nude mice. Am J Pathol 141: $1115-1124$

52. Lockman PR, Mittapalli RK, Taskar KS et al (2010) Heterogeneous blood-tumor barrier permeability determines drug efficacy in experimental brain metastases of breast cancer. Clin Cancer Res 16:5664 5678

53. Percy DB, Ribot EJ, Chen Y, McFadden C, Simedrea C, Steeg PS, Chambers AF, Foster PJ (2011) In vivo characterization of changing blood-tumor barrier permeability in a mouse model of breast cancer metastasis: a complementary magnetic resonance imaging approach. Invest Radiol 46(11):718-725

54. Hermanek P, Hutter RV, Sobin LH, Wittekind C (1999) Classification of isolated tumor cells and micrometastasis. Cancer 86:2668-2673

55. Patchell RA, Tibbs PA, Walsh JW, Dempsey RJ, Maruyama Y, Kryscio RJ, Marksbery WR, Macdonald JS, Young B (1990) A randomized trial of surgery in the treatment of single metastases to the brain. New Engl J Med 322:494-500

56. Patchell RA, Tibbs PA, Regine WF, Dempsey RJ, Mohiuddin M, Kryscio RJ, Markesbery WR, Foon KA, Young B (1998) Postoperative radiotherapy in the treatment of single metastases to the brain-A randomized trial. J Amer Med Assoc 280(17):1485-1489

57. Andrews DW, Scott CB, Sperduto PW, Flanders AE, Gaspar LE, Schell MC, Werner-Wasik M, Demas W, Ryu J, Bahary JP et al (2004) Whole brain radiation therapy with or without stereotactic radiosurgery boost for patients with one to three brain metastases: phase III results of the RTOG 9508 randomised trial. Lancet 363: $1665-1672$

58. Huang F, Alrefae M, Langleben A, Roberge D (2009) Prophylactic cranial irradiation in advanced breast cancer: a case for caution. Int J Radiat Oncol 73(3):752-758 